\title{
Identifying hub genes of papillary thyroid carcinoma in the TCGA and GEO database using bioinformatics analysis
}

\author{
Ying Wan ${ }^{\text {Corresp. Equal first author, } 1}$, Xiaolian Zhang ${ }^{\text {Equal first author, } 2}{ }^{2}$, Huilin Leng ${ }^{3}$, Weihua Yin ${ }^{4}$, Wenxing Zeng ${ }^{1}$, Congling Zhang \\ ${ }^{1}$ Department of Inspection, People's Hospital of Yichun City, Yichun, China \\ 2 Department of Blood Transfusion, People's Hospital of Yichun City, Yichun, China \\ 3 Department of Neurology, People's Hospital of Yichun City, Yichun, China \\ 4 Department of Oncology, People's Hospital of Yichun City, Yichun, China \\ Corresponding Author: Ying Wan \\ Email address: emilywan01@tom.com
}

Background. Thyroid carcinoma (THCA) is a common endocrine malignant tumor. Papillary carcinoma with low degree of malignancy and good prognosis is the most common. It can occur at any age, but it is more common in young adults. Although the mortality rate is decreased due to early diagnosis, the survival rate varies depending on the type of tumor. Therefore, the purpose of this study is to identify hub biomarkers and novel therapeutic targets for THCA.

Methods. The GSE3467, GSE3678, GSE33630 and GSE53157 were obtained from the GEO database, including 100 thyroid tumors and 64 normal tissues to obtain the intersection of differentially expressed genes, and a protein-protein interaction network was constructed to obtain the HUB gene. The corresponding overall survival information from The Cancer Genome Atlas Project-THCA was then included in this research. The signature mechanism was studied by analyzing the gene ontology and the Kyoto Encyclopedia gene and genome database.

Results. In this research, we identified eight candidate genes (FN1, CCND1, CDH2, CXCL12, MET, IRS1, DCN and FMOD) from the network. Also, expression verification and survival analysis of these candidate genes based on TCGA database indicate the robustness of the above results. Finally, our hospital samples validated the expression levels of these genes.

Conclusion. The research identified eight mRNA including four up-regulated and four down-regulated serve as signature, which could be a potential prognostic marker of THCA. 


\title{
1 Identifying hub genes of papillary thyroid carcinoma in the
}

\section{TCGA and GEO database using bioinformatics analysis}

3 Ying Wan ${ }^{1 *}$, Xiaolian Zhang ${ }^{2 *}$, Huilin Leng ${ }^{3}$, Weihua, Yin ${ }^{4}$, Wenxing Zeng ${ }^{1}$, Congling Zhang ${ }^{1}$

41 Department of Inspection, People's Hospital of Yichun City, Yichun, 336000 Jiangxi, China

52 Department of Blood Transfusion, People's Hospital of Yichun City, Yichun, 336000 Jiangxi,

6 China

73 Department of Neurology, People's Hospital of Yichun City, Yichun, 336000 Jiangxi, China

84 Department of Oncology, People's Hospital of Yichun City, Yichun, 336000 Jiangxi, China

* These authors contributed equally to this work.

Corresponding Author:

Ying Wan ${ }^{1}$,

No. 1061 Jinxiu road, Yiyang new district, Yichun, 336000 Jiangxi, China

Email: emilywan01@tom.com

\begin{abstract}
Background.Thyroid carcinoma (THCA) is a common endocrine malignant tumor. Papillary carcinoma with low degree of malignancy and good prognosis is the most common. It can occur at any age, but it is more common in young adults. Although the mortality rate is decreased due to early diagnosis, the survival rate varies depending on the type of tumor. Therefore, the purpose of this study is to identify hub biomarkers and novel therapeutic targets for THCA.

Methods. The GSE3467, GSE3678, GSE33630 and GSE53157 were obtained from the GEO database, including 100 thyroid tumors and 64 normal tissues to obtain the intersection of differentially expressed genes, and a protein-protein interaction network was constructed to obtain the HUB gene. The corresponding overall survival information from The Cancer Genome Atlas Project-THCA was then included in this research. The signature mechanism was studied by analyzing the gene ontology and the Kyoto Encyclopedia gene and genome database.
\end{abstract}

Results. In this research, we identified eight candidate genes (FN1, CCND1, CDH2, CXCL12, MET, IRS1, DCN and FMOD) from the network. Also, expression verification and survival analysis of these candidate genes based on TCGA database indicate the robustness of the above results. Finally, our hospital samples validated the expression levels of these genes.

Conclusion. The research identified eight mRNA including four up-regulated and four downregulated serve as signature, which could be a potential prognostic marker of THCA.

Keywords: thyroid carcinoma, TCGA, GEO, signature, prognosis, biomarker 


\section{Introduction}

Thyroid carcinoma (THCA) is that originates from thyroid tissue and has the potential for distal metastasis (Siegel et al. 2019). It is worth noting that the incidence of women is two to four times higher than that of men (Carling \& Udelsman 2014). THCA is mainly divided into four categories, including papillary carcinoma (85\%), follicular carcinoma (10-15\%), medullary carcinoma $(5-10 \%)$ and undifferentiated THCA $(<5 \%)$. As a result of early diagnosis, the mortality rate of THCA is stable or declining, although the incidence of THCA continues to rise (Wang et al. 2019b). Notwithstanding, the high incidence of papillary thyroid cancer (PTC) is depended on many uncontrollable factors, such as specific genetic changes of THCA and traumatic invasive examination has different degrees of damage to the patient's thyroid. Therefore, it is very important to identify hub biomarker and non-invasive methods for PTC.

With the development of RNA sequencing and microarray technology, the study of differentially expressed gene (DEG) between cancer and normal tissues has improved our understanding of the molecular mechanism of PTC. It also could help us identify important biomarkers. Previous studies were based on one dataset with limited sample size, and it is not the best way to get DEGs from a single dataset (Liang \& Sun 2018; Zhao et al. 2016). Above defects may lead to a deviation in the final result.

In this research, we conducted a multi-step analysis through comprehensive bioinformatics analysis to identify hub genes in THCA. We obtained the expression profiles of GSE3467, GSE3678, GSE33630 and GSE53157 with PTC from the GEO database, which included a total of 100 THCA and 64 normal samples. After the intersection, we got a total of 179 DEGs in four datasets. Then, we carried out above genes through bioinformatics analysis, which provided reliable and novel biomarkers for THCA, which will be helpful for further clinical application in the diagnosis, prognosis and targeted treatment of PTC.

\section{Methods}

Datasets selecting and DEGs identification

We downloaded the four gene expression datasets of PTC from GEO database, including the following criterias: (a) PTC, (b) datasets including tumor and normal tissues, (c) the organism is Homo sapiens, (d) Sample size exceeding 10 samples. GSE3467 ( $\mathrm{n}=18)$, GSE3678 ( $\mathrm{n}=14)$, GSE33630 ( $n=60)$ and GSE53157 ( $=27)$ were among depended on the GPL570 platform. The limma package was used to identify the DEGs in each GEO datasets in R (Version 3.7.1) (Ritchie et al. 2015). The $P$-value is determined by the false discovery rate. The standard for DEG is that the false discovery rate is less than 0.05 , and the criteria of the groups were $\mid \log _{2} \mathrm{FC}$ (fold change) $\mid \geqslant 1$.

KEGG and GO enrichment analyses of DEGs

To explore biological information and obtain more comprehensive gene and protein functions, we used clusterProfile packages for GO, which including cellular component (CC) biological 
process (BP) and molecular function (MF), and KEGG analysis (Yu et al. 2012).

PPI network construction

The online database is used to predict the PPI network, which is used to retrieve the interacting genes (STRING; http://string-db.org) (Szklarczyk et al. 2017). In this research, the PPI network of genes was established with STRING database, and the interaction with high confidence $>0.4$ was statistically significant. Next, we use Cytoscape (Version 3.6.1), a free bioinformatics software, to visualise and analyse the molecular interaction networks. TCGA database validation and survival analysis

In order to verify the results of the hub genes, we also obtained the gene expression profiles from The Cancer Genome Atlas (TCGA) (https://cancergenome.nih.gov/) database to validate them, including 502 tumor and 58 non-tumor samples. SurvExpress, a free online website, uses log-rank tests to draw Kaplan-Meier plots that contain the "Survival" package of R software. Clinical patient samples

A total of 39 patients with THCA diagnosed in People's Hospital of Yichun City from October 2018 to August 2019 were included in this study. This study was approved by the Ethical Committee of People's Hospital of Yichun City (PHYC-2018-10-8-1). All patients had not received any radiotherapy or chemotherapy before sampling and signed a written informed consent form. Immediately after surgical resection, the tumor and adjacent non-tumor tissues

were transferred to liquid nitrogen and stored in the refrigerator at $-80{ }^{\circ} \mathrm{C}$ until RNA was extracted.

RNA extraction and qRT-PCR analysis

Total RNA was extracted by TRIzol reagent (Invitrogen, CA, USA) from tissues. After dissolution, NanoDrop Lite spectrophotometer to evaluate the concentration and purity of total RNA. Then, the total RNA reverse transcription synthesis of cDNA and used to conduct qRTPCR assays for genes. GAPDH was used as internal control, and PCR experiments were repeated three times. Fold change $\left(2^{-\Delta \Delta \mathrm{CT}}\right)$ was represented relative gene expression levels. Table 1 was listed all the primer sequences and small interfernce sequences, and divergent primer was designed for each gene.

\section{Results}

Intersection of DEGs in four GEO datasets

As shown in Figure. 1, we conducted a multi-step analysis to study hub DEGs and its important biological functions and prognosis in THCA through comprehensive bioinformatics methods. To begin with, we chose and obtained a total of four gene expression profiles of THCA from GEO datasets (GSE3467, GSE3678, GSE33630 and GSE53157). In this research, 100 cases of thyroid tumors and 64 cases of normal tissues were obtained.

The DEG distribution in every GEO datasets demonstrated in the form of volcano plots (Figure. 2a-d), including 851 DEGs in GSE3467, 785 DEGs in GSE3678, 1404 DEGs in GSE33630, and 858 DEGs in GSE53157. The intersection of 179 DEGs in four GEO datasets 
115 was displayed in Figure. 2e.

116 Functional annotation of DEGs

117 GO enrichment analysis was carried out to research the biological functions of all the DEGs.

118 The BP classification of GO analysis demonstrated that the DEGs obviously enriched the

119 skeletal system development, muscle cell differentiation and regulation of chromosome

120 organization. For CC, these DEGs are rich in chromatin, adherens junction and nuclear

121 chromosome part. In addition they are significantly rich in transcription coregulator activity,

122 proximal promoter sequence-specific DNA binding and RNA polymerase II proximal from

123 promoter in the MF category (Figure. 3a).

124 The enrichment analysis of KEGG pathway displayed that DEGs were obviously abundant in

proteoglycans in cancer (Figure. 3b). Pathways of PTC were also contained, including metabolic pathways, neuroactive ligand-receptor interaction, rap1 signaling pathway, thyroid hormone signaling pathway.

Construction of PPI network

In order to determine the interaction of these DEGs in THCA, we used the STRING database to build a PPI network in Cytoscape software (Figure. 4a), which included 127 nodes and 199 edges. Next, we use the plug-in cytoHubba in Cytoscape to screen out the first eight hub genes. They are FN1, CCND1, CDH2, CXCL12, MET, IRS1, DCN and FMOD using the MCC method in Figure $4 b$.

Prognostic gene screening

In order to verify above results of all the DEGs and candidate genes obtained in other datasets, we obtained the gene expression profiles of all 179 DEGs in THCA from the TCGA database. Additionally, a distinctly different expression pattern between the tumor and normal samples were found (Figure. 5a-h). We explored the association between gene expression and survival, and found that only DCN was significantly correlated with the overall survival (OS) of THCA samples trough the GEPIA database (log-rank $p=0.01892$, Figure 5i).

Validation of differentially expressed levels for hub genes by qRT-PCR

The levels of eight hub genes expression (FN1, CCND1, CDH2, CXCL12, MET, IRS1, DCN and FMOD) in PTC and para-cancerous non-tumor tissues were detected by qRT-PCR. As shown in Figure. 6, FN1 (fibronectin 1), CCND1 (Cyclin D1), CDH2 (cadherin 2), MET (MET proto-oncogene, receptor tyrosine kinase), were significantly upregulated in THCA tissues $(\mathrm{n}=$ 87) compared with normal samples. Additionally, IRS1 (insulin receptor substrate 1), DCN (decorin), FMOD (fibromodulin), and CXCL12 (chemokine C-X-C motif ligand 12) were downregulated in tumor tissues. The above results confirmed the accuracy of our analysis and showed that these eight key genes can be considered as a potential signature or biomarker of THCA.

\section{Discussion}

In the present study, we screened 179 robust DEGs in PTC's four GEO datasets. These DEGs were obviously enriched in a variety of cancer-related functions and pathways by GO and KEGG 
155

156

157

158

159

160

pathway enrichment analysis. Use the STRING database to build the PPI network, and carry on the hub genes. We finally screened eight central genes from the whole network, including FN1, CCND1, CDH2, CXCL12, MET, IRS1, DCN and FMOD, which genes are involved in the regulation of cyclin gene or cadherin gene, and could participate in the development of PTC. The expression verification and prognosis analysis of these candidate genes depended on TCGA database showed the robustness and reliability of the above results.

Several studies had been published about DEGs in human malignant tumors, such as THCA. Liu et al. identified 359 DEGs between tumor and normal thyroid tissues from TCGA database (Liu et al. 2019). Among them, five DEGs (LPAR5, NMU, FN1, NPY1R, and CXCL12) were screened with higher degrees and LPAR5 was associated with OS. Compared with our research, among the top eight hub genes (FN1, CCND1, CDH2, CXCL12, MET, IRS1, DCN and FMOD), we found that only DCN existed significance of OS in this study.

Compared with previously published researches on THCA, our research fully integrates multiple databases, datasets, and large sample sizes, including tumors and normal tissues. The results we got were robust and powerful, which could have non-invasive examination for patients with PTC.

Through palpation, doctors can find thyroid nodules, which are hard in texture and not smooth on the surface. At the same time, if the nodules do not move with the trachea when swallowing, and the enlarged and hard lymph nodes in the neck can be touched, thyroid cancer should be taken into account. If the tumor is in a small stage, ultrasound has become a very good screening tool for thyroid space-occupying lesions, which is widely used in thyroid physical examination and clinical differential diagnosis. However, the ultrasonic features of benign and malignant nodules overlap, and ultrasound doctors' interpretations and suggestions on these features are quite different in clinical practice, which requires further fine needle puncture and genetic testing at the same time. We obtained eight hub genes in the network, which can be used for unique expression levels in PTC, and we can even diagnose PTC in early diagnosis. Some of which have been proved to be associated with PTC, depended on PPI network and candidate genes evaluation. Low fibronectin 1 (FN1) expression in tumorous tissues was an independent worse prognostic factor for progression-free survival in sporadic medullary thyroid carcinoma (Zhan et al. 2018). Additionally, Ouchi et al. identified FN1 as a fusion partner of ALK by 5'RACE, FN1ALK, resulted in ALK overexpression in the inflammatory myofibroblastic tumors, and this finding should facilitate development of novel therapeutics (Ouchi et al. 2015). Also, FN1 could encode fibronectin, which was associated with various cancers precession, including cervical cancer (Wang et al. 2019a), gastric cancer (Jiang et al. 2019), colorectal cancer (Wu et al. 2016). Cyclin D1 (CCND1) functions as regulators of CDK kinases, and forms a complex with and functions as a regulatory subunit of CDK4 or CDK6, whose activity is required for cell cycle $\mathrm{G} 1 / \mathrm{S}$ transition. It was proven as a DEG and used as a diagnostic and predictive biomarker in thyroid carcinoma and may be functionally involved in the development and progression of the disease (Jeon et al. 2018). In addition, down regulation of CCND1 may be an important mechanism by which everolimus increases the therapeutic window of paclitaxel in cervical cancers (Yilmaz et al. 2016). The biological role of cadherin 2 (CDH2) was also mostly reported 
196

197

198

199

200

201

202

203

204

205

206

207

208

209

210

211

212

213

214

215

216

217

218

219

220

221

222

223

224

225

226

227

228

229

230

231

232

233

234

235

in glioma with prognostic significance (Chen et al. 2018b). Previous studies showed that chemokine (C-X-C motif) ligand 12 (CXCL12) had reported in different human tumors. CXCL12 could activate CXCR4 as well as multiple downstream multiple tumorigenic signaling pathways, promoting expression of various oncogenes. Activation of the CXCL12-CXCR4 signaling axis promotes epithelial-mesenchymal transition (EMT) (Sleightholm et al. 2017; Yang et al. 2019). The biological roles of CXCL12 also were founded in THCA, in which CXCR4/CXCR7/CXCL12 axis offer new valuable insight into the oncogenesis of metastatic follicular thyroid carcinoma (Werner et al. 2018). The amplification of MET proto-oncogene, receptor tyrosine kinase (MET) is confirmed as a resistance factor in gastric cancer cells (Ebert et al. 2019). And, MET may serve a role in regulating PD-L1 expression in hepatocellular carcinoma (Chun \& Hong 2019) Meanwhile, MET is also involved in high-risk metastasis progression in thyroid cancer (Garcia et al. 2019). Insulin receptor substrate 1 (IRS1) can be phosphorylated to activate AKT kinase, which promotes the development of lung cancer (Gorgisen et al. 2019). The low expression of decorin (DCN) has been demonstrated to contribute to be as a potential biomarker in colon cancer (Li et al. 2017). DCN was found underexpressed in thyroid tumors (Arnaldi et al. 2005). Qian et al. had shown that the methylated $+58 \mathrm{CpG}$ in DCN 5'-UTR involved in reduced the mRNA expression of DCN (Qian et al. 2014). In our research, Fibromodulin (FMOD) acted as an important mediator in VEGF expression and angiogenesis induced by GDNF in human glioblastoma (Chen et al. 2018a).

The expression of DCN gene in cancer tissues was lower than that in para-cancerous tissues. However, the lower the expression in cancer tissue, the better the prognosis was. This DCN is still an oncogene, but its pathway may be suppressed as a whole, with low expression or low levels of other regulation. It can still have an effect on tumor growth, but it is not the main driving gene. This can also happen if the prognosis of the tumor is good.

However, functional experiments should be carried out to verify our results. In addition, further research on a larger sample size will be required.

\section{Conclusion}

We found eight hub genes including four up-regulated and four down-regulated in PTC, and disclosed that DCN gene is an important gene related to prognosis through TCGA database to verify the accuracy of our finding. This hub genes may be considered as a new and potential signature or biomarker of PTC, which will further aid in contributing to the diagnosis, prognosis and clinical application of therapeutic targets for PTC.

Arnaldi LA, Borra RC, Maciel RM, and Cerutti JM. 2005. Gene expression profiles reveal that DCN, DIO1, and DIO2 are underexpressed in benign and malignant thyroid tumors. Thyroid 15:210-221. 10.1089/thy.2005.15.210

Carling T, and Udelsman R. 2014. Thyroid cancer. Annu Rev Med 65:125-137. 10.1146/annurev-med-061512105739 
236

237

238

239

240

241

242

243

244

245

246

247

248

249

250

251

252

253

254

255

256

257

258

259

260

261

262

263

264

265

266

267

268

269

270

271

272

273

274

275

276

Chen M, Ba H, Lu C, Dai J, and Sun J. 2018a. Glial Cell Line-Derived Neurotrophic Factor (GDNF) Promotes Angiogenesis through the Demethylation of the Fibromodulin (FMOD) Promoter in Glioblastoma. Med Sci Monit 24:6137-6143. 10.12659/MSM.911669

Chen Q, Cai J, and Jiang C. 2018b. CDH2 expression is of prognostic significance in glioma and predicts the efficacy of temozolomide therapy in patients with glioblastoma. Oncol Lett 15:7415-7422. 10.3892/ol.2018.8227

Chun HW, and Hong R. 2019. Significance of PD-L1 clones and C-MET expression in hepatocellular carcinoma. Oncol Lett 17:5487-5498. 10.3892/ol.2019.10222

Ebert K, Mattes J, Kunzke T, Zwingenberger G, and Luber B. 2019. MET as resistance factor for afatinib therapy and motility driver in gastric cancer cells. PLoS One 14:e0223225. 10.1371/journal.pone.0223225

Garcia C, Buffet C, El Khattabi L, Rizk-Rabin M, Perlemoine K, Ragazzon B, Bertherat J, Cormier F, and Groussin L. 2019. MET overexpression and activation favors invasiveness in a model of anaplastic thyroid cancer. Oncotarget 10:2320-2334. 10.18632/oncotarget.26798

Gorgisen G, Hapil FZ, Yilmaz O, Cetin Z, Pehlivanoglu S, Ozbudak IH, Erdogan A, and Ozes ON. 2019. Identification of novel mutations of Insulin Receptor Substrate 1 (IRS1) in tumor samples of non-small cell lung cancer (NSCLC): Implications for aberrant insulin signaling in development of cancer. Genet Mol Biol 42:15-25. 10.1590/1678-4685-gmb-2017-0307

Jeon S, Kim Y, Jeong YM, Bae JS, and Jung CK. 2018. CCND1 Splice Variant as A Novel Diagnostic and Predictive Biomarker for Thyroid Cancer. Cancers (Basel) 10. 10.3390/cancers10110437

Jiang K, Liu H, Xie D, and Xiao Q. 2019. Differentially expressed genes ASPN, COL1A1, FN1, VCAN and MUC5AC are potential prognostic biomarkers for gastric cancer. Oncol Lett 17:3191-3202. 10.3892/ol.2019.9952

Li G, Li M, Liang X, Xiao Z, Zhang P, Shao M, Peng F, Chen Y, Li Y, and Chen Z. 2017. Identifying DCN and HSPD1 as Potential Biomarkers in Colon Cancer Using 2D-LC-MS/MS Combined with iTRAQ Technology. J Cancer 8:479-489. 10.7150/jca.17192

Liang W, and Sun F. 2018. Identification of key genes of papillary thyroid cancer using integrated bioinformatics analysis. J Endocrinol Invest 41:1237-1245. 10.1007/s40618-018-0859-3

Liu L, He C, Zhou Q, Wang G, Lv Z, and Liu J. 2019. Identification of key genes and pathways of thyroid cancer by integrated bioinformatics analysis. J Cell Physiol. 10.1002/jcp.28932

Ouchi K, Miyachi M, Tsuma Y, Tsuchiya K, lehara T, Konishi E, Yanagisawa A, and Hosoi H. 2015. FN1: a novel fusion partner of ALK in an inflammatory myofibroblastic tumor. Pediatr Blood Cancer 62:909-911. 10.1002/pbc. 25424

Qian Q, Shi X, Lei Z, Zhan L, Liu RY, Zhao J, Yang B, Liu Z, and Zhang HT. 2014. Methylated +58CpG site decreases DCN mRNA expression and enhances TGF-beta/Smad signaling in NSCLC cells with high metastatic potential. Int J Oncol 44:874-882. 10.3892/ijo.2014.2255

Ritchie ME, Phipson B, Wu D, Hu Y, Law CW, Shi W, and Smyth GK. 2015. limma powers differential expression analyses for RNA-sequencing and microarray studies. Nucleic Acids Res 43:e47. 10.1093/nar/gkv007

Siegel RL, Miller KD, and Jemal A. 2019. Cancer statistics, 2019. CA Cancer J Clin 69:7-34. 10.3322/caac.21551

Sleightholm RL, Neilsen BK, Li J, Steele MM, Singh RK, Hollingsworth MA, and Oupicky D. 2017. Emerging roles of the CXCL12/CXCR4 axis in pancreatic cancer progression and therapy. Pharmacol Ther 179:158-170. 10.1016/j.pharmthera.2017.05.012

Szklarczyk D, Morris JH, Cook H, Kuhn M, Wyder S, Simonovic M, Santos A, Doncheva NT, Roth A, Bork P, Jensen L, and von Mering C. 2017. The STRING database in 2017: quality-controlled protein-protein association

Peer) reviewing PDF | (2019:11:43261:4:0:NEW 9 Apr 2020) 
networks, made broadly accessible. Nucleic Acids Res 45:D362-D368. 10.1093/nar/gkw937

Wang S, Gao B, Yang H, Liu X, Wu X, and Wang W. 2019a. MicroRNA-432 is downregulated in cervical cancer and directly targets FN1 to inhibit cell proliferation and invasion. Oncol Lett 18:1475-1482. 10.3892/ol.2019.10403

Wang Y, Huang H, Hu F, Li J, Zhang L, and Pang H. 2019b. CITED1 contributes to the progression of papillary thyroid carcinoma via the Wnt/beta-catenin signaling pathway. Onco Targets Ther 12:6769-6777. 10.2147/OTT.S215025

Werner TA, Forster CM, Dizdar L, Verde PE, Raba K, Schott M, Knoefel WT, and Krieg A. 2018. CXCR4/CXCR7/CXCL12-Axis in Follicular Thyroid Carcinoma. J Cancer 9:929-940. 10.7150/jca.23042

Wu J, Wang Y, Xu X, Cao H, Sahengbieke S, Sheng H, Huang Q, and Lai M. 2016. Transcriptional activation of FN1 and IL11 by HMGA2 promotes the malignant behavior of colorectal cancer. Carcinogenesis 37:511-521. 10.1093/carcin/bgw029

Yang P, Hu Y, and Zhou Q. 2019. The CXCL12-CXCR4 signaling axis plays a key role in cancer metastasis and is a potential target for developing novel therapeutics against metastatic cancer. Curr Med Chem. 10.2174/0929867326666191113113110

Yilmaz A, Alp E, Onen HI, and Menevse S. 2016. Reduced BCL2 and CCND1 mRNA expression in human cervical cancer HeLa cells treated with a combination of everolimus and paclitaxel. Contemp Oncol (Pozn) 20:2832. $10.5114 /$ wo. 2016.58498

Yu G, Wang LG, Han Y, and He QY. 2012. clusterProfiler: an R package for comparing biological themes among gene clusters. OMICS 16:284-287. 10.1089/omi.2011.0118

Zhan S, Li J, Wang T, and Ge W. 2018. Quantitative Proteomics Analysis of Sporadic Medullary Thyroid Cancer Reveals FN1 as a Potential Novel Candidate Prognostic Biomarker. Oncologist 23:1415-1425. 10.1634/theoncologist.2017-0399

Zhao M, Wang KJ, Tan Z, Zheng CM, Liang Z, and Zhao JQ. 2016. Identification of potential therapeutic targets for papillary thyroid carcinoma by bioinformatics analysis. Oncol Lett 11:51-58. 10.3892/ol.2015.3829 
Table $\mathbf{1}$ (on next page)

Table 1 mRNA primers 
1 Table1 mRNA PCR primer

\begin{tabular}{|l|l|}
\hline Gene name & \multicolumn{1}{|c|}{ Primer sequence } \\
\hline \multirow{2}{*}{ FN1 } & F: CGGTGGCTGTCAGTCAAAG \\
\cline { 2 - 2 } & R: AAACCTCGGCTTCCTCCATAA \\
\hline \multirow{2}{*}{ CCND1 } & F: GCTGCGAAGTGGAAACCATC \\
\cline { 2 - 2 } & R: CCTCCTTCTGCACACATTTGAA \\
\hline \multirow{2}{*}{ CDH2 } & F: TCAGGCGTCTGTAGAGGCTT \\
\cline { 2 - 2 } & R: ATGCACATCCTTCGATAAGACTG \\
\hline \multirow{2}{*}{ CXCL12 } & F: ATTCTCAACACTCCAAACTGTGC \\
\cline { 2 - 2 } & R: ACTTTAGCTTCGGGTCAATGC \\
\hline \multirow{2}{*}{ MET } & F: AGCAATGGGGAGTGTAAAGAGG \\
\cline { 2 - 2 } & R: CCCAGTCTTGTACTCAGCAAC \\
\hline \multirow{2}{*}{ IRS1 } & F: ACAAACGCTTCTTCGTACTGC \\
\cline { 2 - 2 } & R: AGTCAGCCCGCTTGTTGATG \\
\hline \multirow{2}{*}{ DCN } & F: ATGAAGGCCACTATCATCCTCC \\
\cline { 2 - 3 } & R: GTCGCGGTCATCAGGAACTT \\
\hline \multirow{2}{*}{ FMOD } & F: GAGACCTACGAGCCTTACCC \\
\cline { 2 - 2 } & R: TTGAGGTTGCGATTGTCACAG \\
\hline
\end{tabular}

2 


\section{Figure 1}

Figure_1

The development and verification process of the assay method is summarized and detailed in the following sections. Gene Expression Omnibus; DEG, differentially expressed genes; PPI, protein-protein interaction; TCGA, The Cancer Genome Atlas 


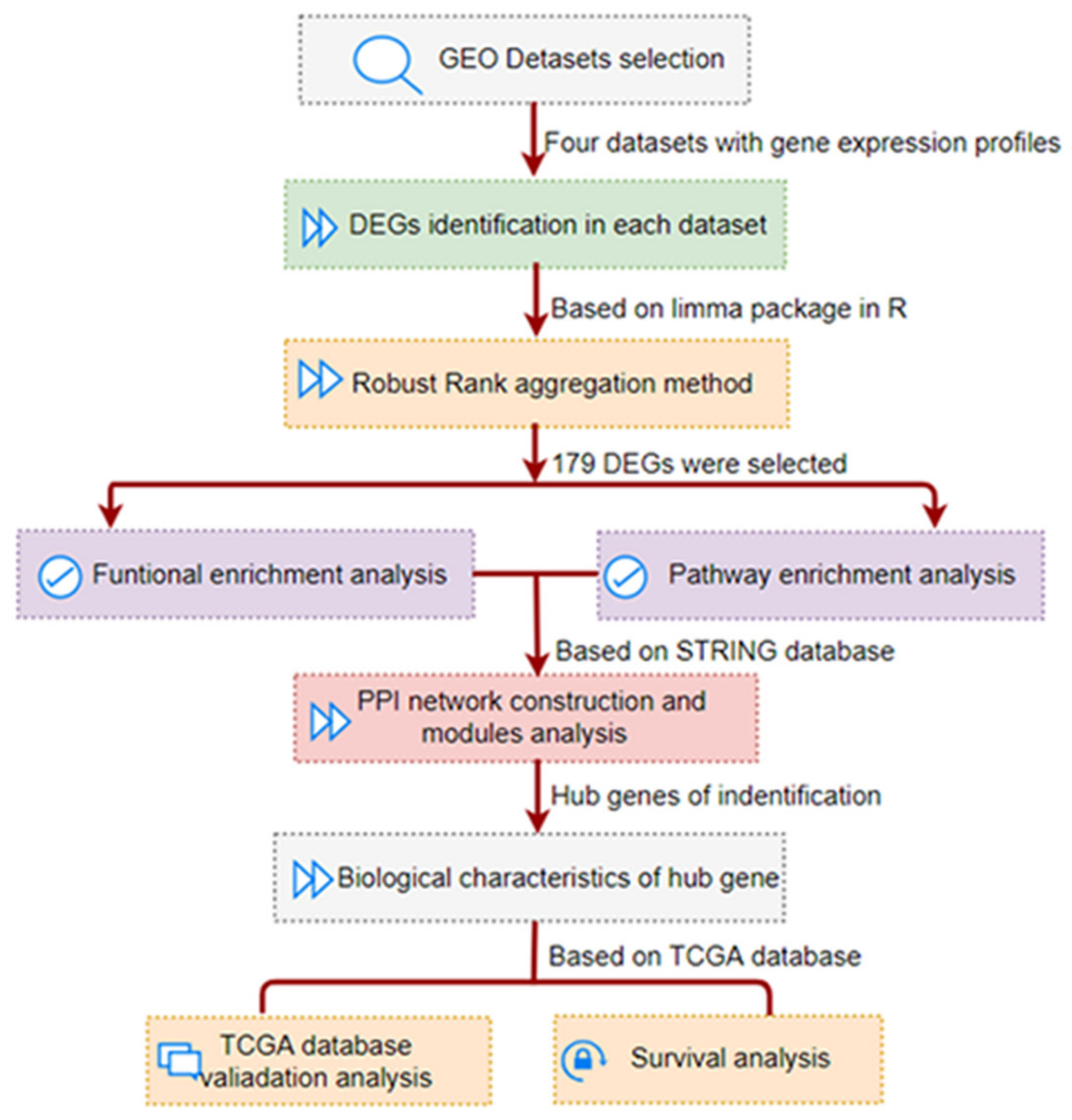




\section{Figure 2}

Figure_2

Filter and identify DEGs in various GEO datasets. (a - d) The volcanic map of the DEGs distribution was shown in GSE3467 (a), GSE3678 (b), GSE33630 (c) and GSE53157 (d). (e) Intersection of four datasets. DEG, differentially expressed gene
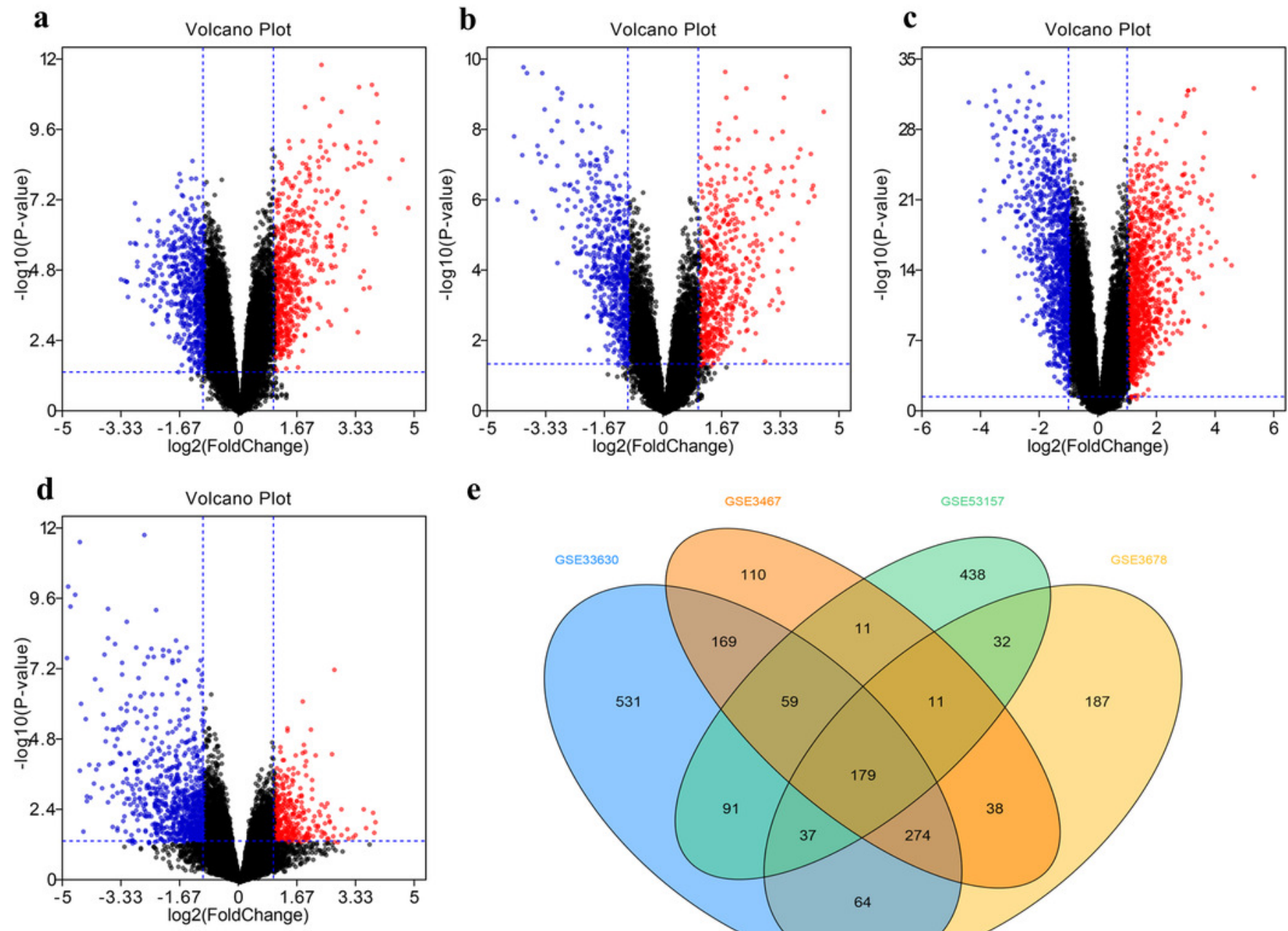

e

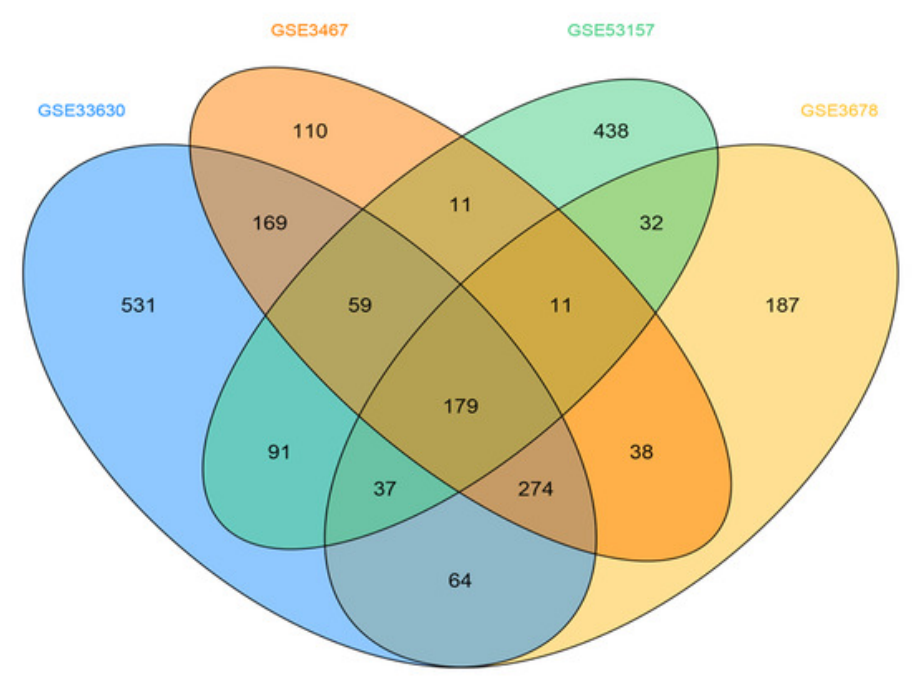




\section{Figure 3}

Figure_3

Gene Ontology and Kyoto Encyclopaedia of Genes and Genomes pathway enrichment analyses. (a) Genes were assigned to GO categories and the terms were summarized into three main GO categories. (b) Enrichment Analysis of abnormal regulation DEGs in Bubble Diagram. DEG, differentially expressed gene. 
$\mathbf{a}$

\section{The Most Enriched GO Terms}

mesenchyme development nuclear-transcribed mRNA poly(A) tail... cardiac epithelial to mesenchymal. mesenchymal cell differentiation endocardial cushion development muscle cell differentiation cardiac septum development heart morphogenesis -

stem cell population maintenance regulation of chromosome organization maintenance of cell number sister chromatid cohesion skeletal system development mesenchyme morphogenesis visual system development nuclear chromatin -

synaptic vesicle membrane exocytic vesicle membrane nuclear chromosome part exocytic vesicle ruffle synaptic vesicle -

transcriptional repressor complex DNA-binding transcription activator acti... proximal promoter sequence-specific DNA... beta-catenin binding ubiquitin-like protein transferase activ..

RNA polymerase II proximal..

transcription coregulator activity Ras GTPase binding DNA-binding transcription repressor acti... small GTPase binding mitogen-activated protein kinase kinase..

ubiquitin-like protein ligase activity transmembrane receptor protein kinase... protein phosphatase binding -

ubiquitin-protein transferase activity adenylyltransferase activity

\section{b}

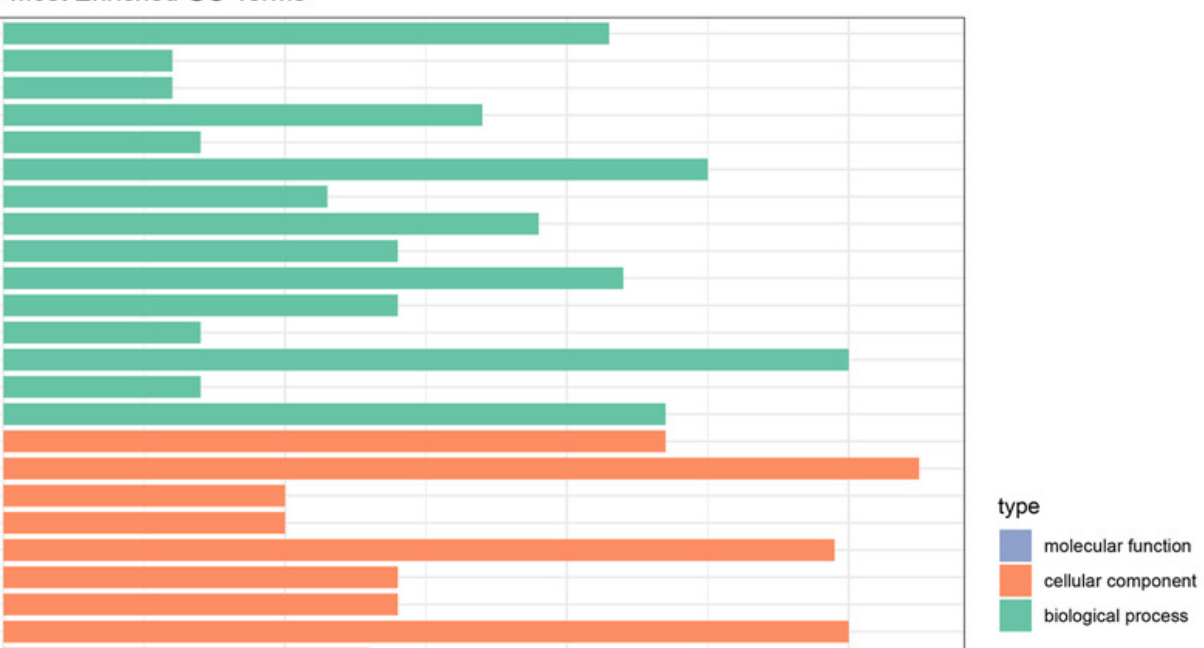

Melanoma
Spliceosom

Chronic myeloid leukemia Small cell lung cancer TGF-beta signaling pathway

Cell cycle

Epstein-Barr virus infection Viral carcinogenesis

Protein processing in endoplasmic reticulum Toxoplasmosis

Transcriptional misregulation in cancer Hepatitis B

FoxO signaling pathway HTLV-I infection Apoptosis Endocytosis Signaling pathways regulating pluripotency of stem cells cGMP-PKG signaling pathway PI3K-Akt signaling pathway Hippo signaling pathway Axon guidance Jak-STAT signaling pathway MicroRNAs in cancer Influenza A

Ras signaling pathway Focal adhesion

Pathways in cancer MAPK signaling pathway cAMP signaling pathway

Metabolic pathways
Inputnumber

- 10

- 20

- 30

PValue

0.010

0.005 
Figure 4

Figure_4

PPI of DEGs. (a) The red nodes represent the hub genes. (b) A redder node means a higher degree.

a

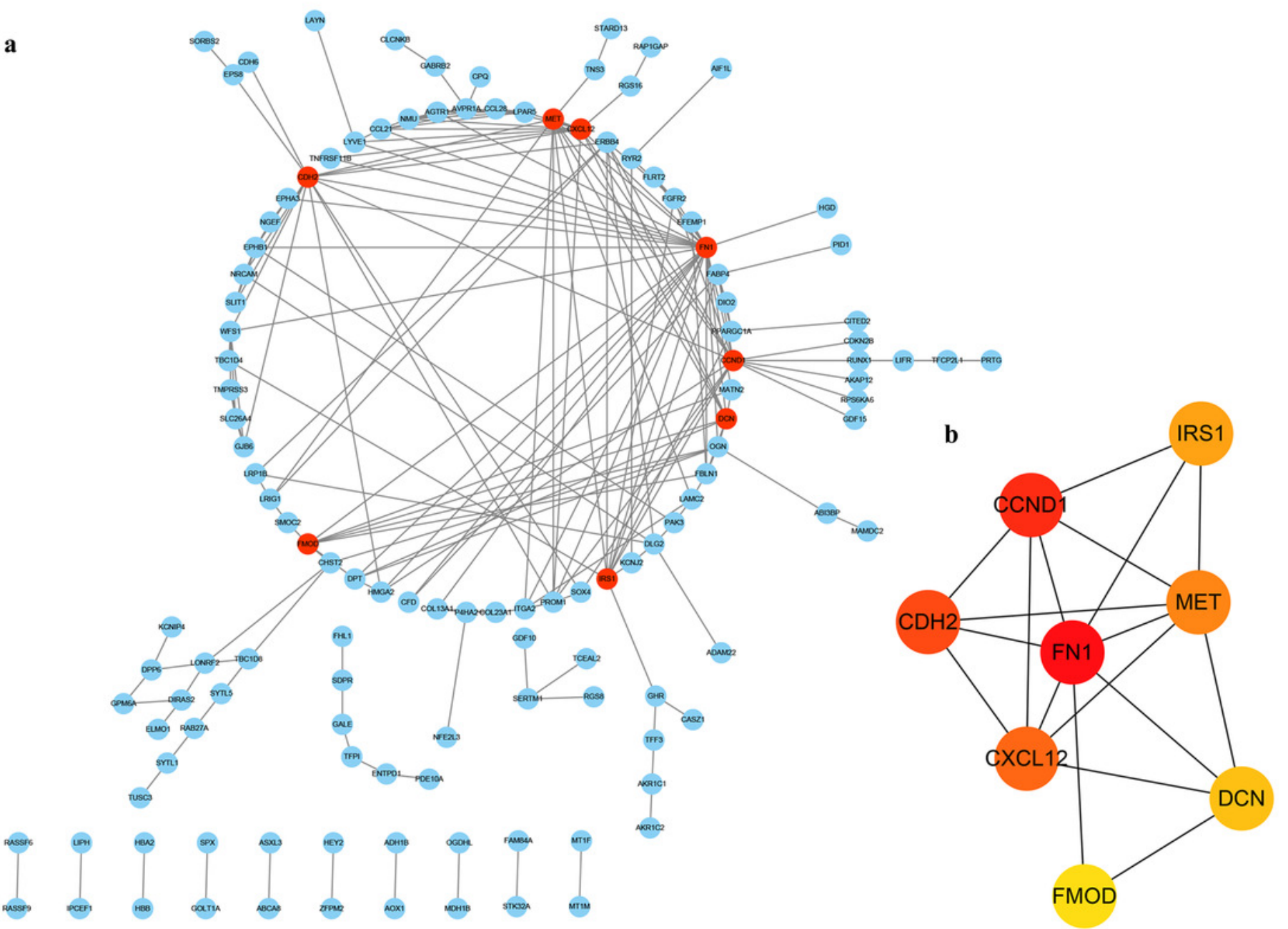


Figure 5

Figure_5

The expression of genes on TCGA. (a) FN1. (b) CCND1 (c) CDH2 (d) CXCL12 (e) MET (f) IRS1 (g) DCN (h) FMOD (i) The OS of DCN in TCGA database. Red represent cancer tissues, grey represent normal tissues. * means $\mathrm{P}<0.05$. TCGA, The Cancer Genome Atlas; OS, overall survival.
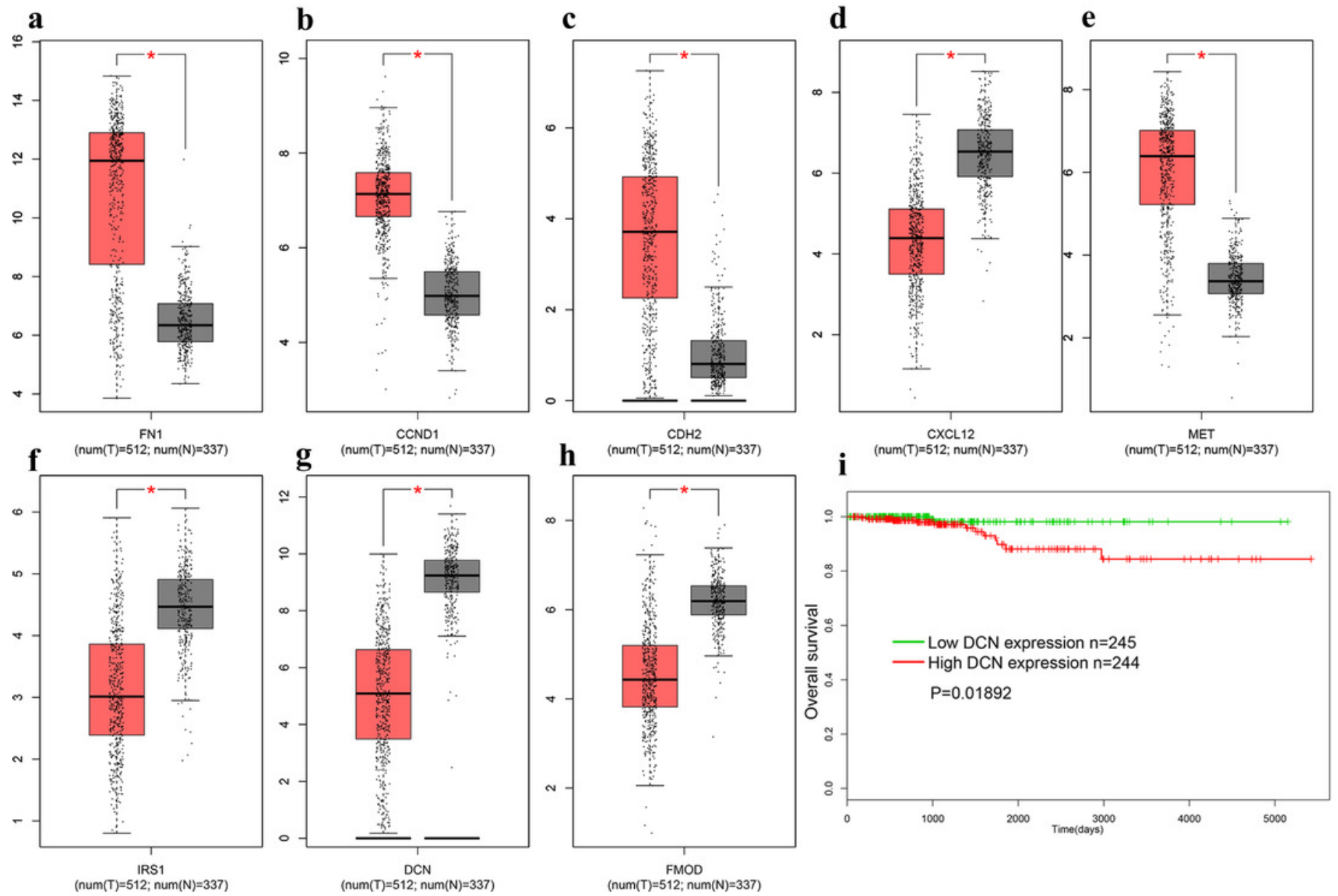
Figure 6

Figure_6

Relative expression levels of hub genes detected by qRT-PCR. ${ }^{*} p<0.05, * * p<0.01$ and ${ }^{* * *} p$ $<0.001$. (a) FN1. (b) CCND1 (c) CDH2 (d) CXCL12 (e) MET (f) IRS1 (g) DCN (h) FMOD. qRT$\mathrm{PCR}$, quantitative real time polymerase chain reaction
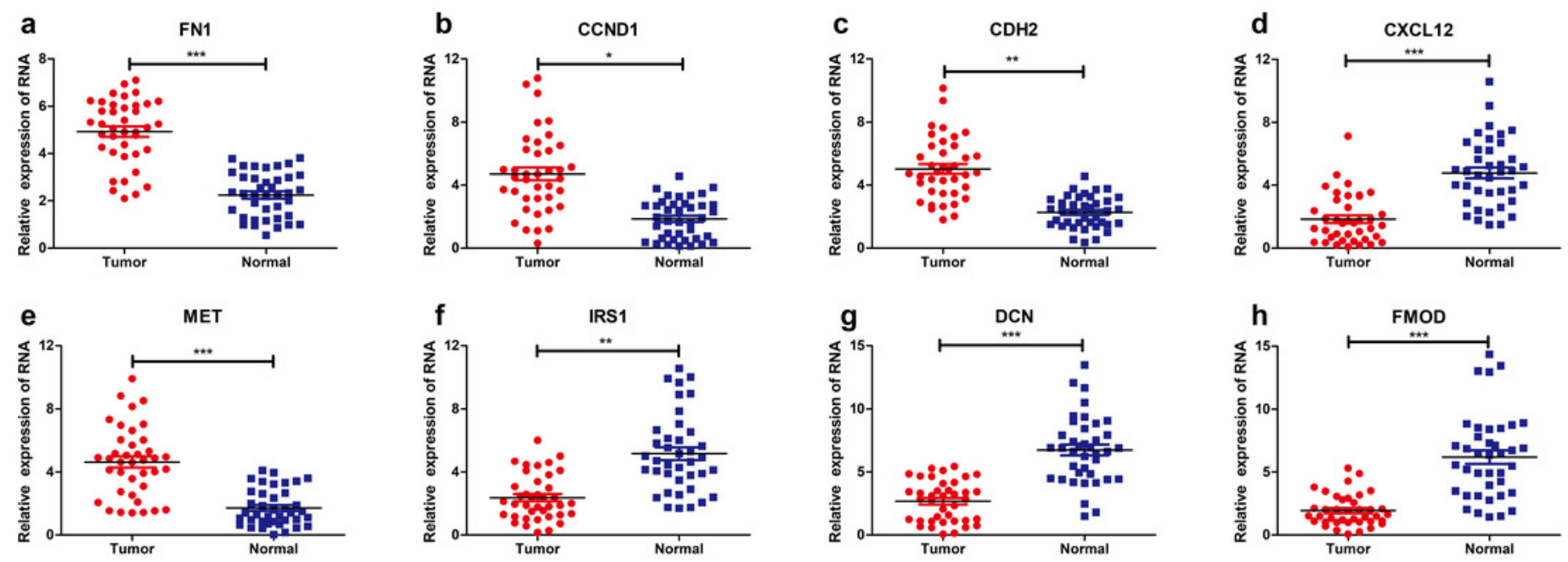